\title{
Reinforcement Learning for Context Aware Segmentation
}

\author{
Lichao Wang, Robert Merrifield, and Guang-Zhong Yang \\ The Hamlyn Centre for Robotic Surgery, Imperial College London, UK \\ \{lichao.wang, rdm99, g.z.yang\}@imperial.ac.uk
}

\begin{abstract}
The ability to learn from user behavior during image segmentation to replicate the innate human ability to adapt shape delineation to contextually specific local information is an important area of study in image understanding. Current approaches to image segmentation usually incorporate specific designs, either relying on generic image features or specific prior knowledge, which usually prevent their application in different contextual settings. In this paper, a general segmentation framework based on reinforcement learning is proposed. It demonstrates how user-specific behavior can be assimilated in-situ for effective model adaptation and learning. It incorporates a two-layer reinforcement learning algorithm that constructs the model from accumulated experience during user interaction. As the algorithm learns 'pervasively' whilst the user performs manual segmentation, no additional steps are required for the training process, allowing the method to adapt and improve its accuracy as experience is acquired. Detailed validation of the method on in-vivo magnetic resonance (MR) data demonstrates the practical value of the technique in significantly reducing the level of user interaction required, whilst maintaining the overall segmentation accuracy.
\end{abstract}

\section{Introduction}

Automated image segmentation for medical imaging is inherently challenging due to the complexity of anatomical structures and inevitable artefacts introduced during data acquisition. Current approaches can be divided into two main categories: methods relying on generic image features and those combining prior knowledge, whether being geometrical or statistical, with specific image content being examined. Active contours and level-set methods [1,2] are typical approaches focusing on the current image content, whereas Statistical Shape Models (SSM) [3] instantiate the overall behavior of a group of shapes on a specific data set. Techniques such as SSM are heavily dependent on a comprehensive training set with residual issues of over-fitting and poor generalisation to pathological data in clinical application [4-6]. Thus far, limited attention is paid to the learning of user behavior during manual segmentation. It has long been recognised that experienced observers have innate abilities in adapting shape delineation to local information that is contextually specific. Fig. 1 illustrates three typical examples of image segmentation showing (a) the endocardial border of the left ventricle (LV), where users do not always rely on gradient features for myocardial mass measurement when papillary muscles are excluded; (b) the 
avoidance of the partial volume effect of MR; and (c) bridging across both subtle and strong anatomical boundaries during the segmentation of the inner and outer contours of the levator ani, the tripartite muscle sheet of the pelvic floor.

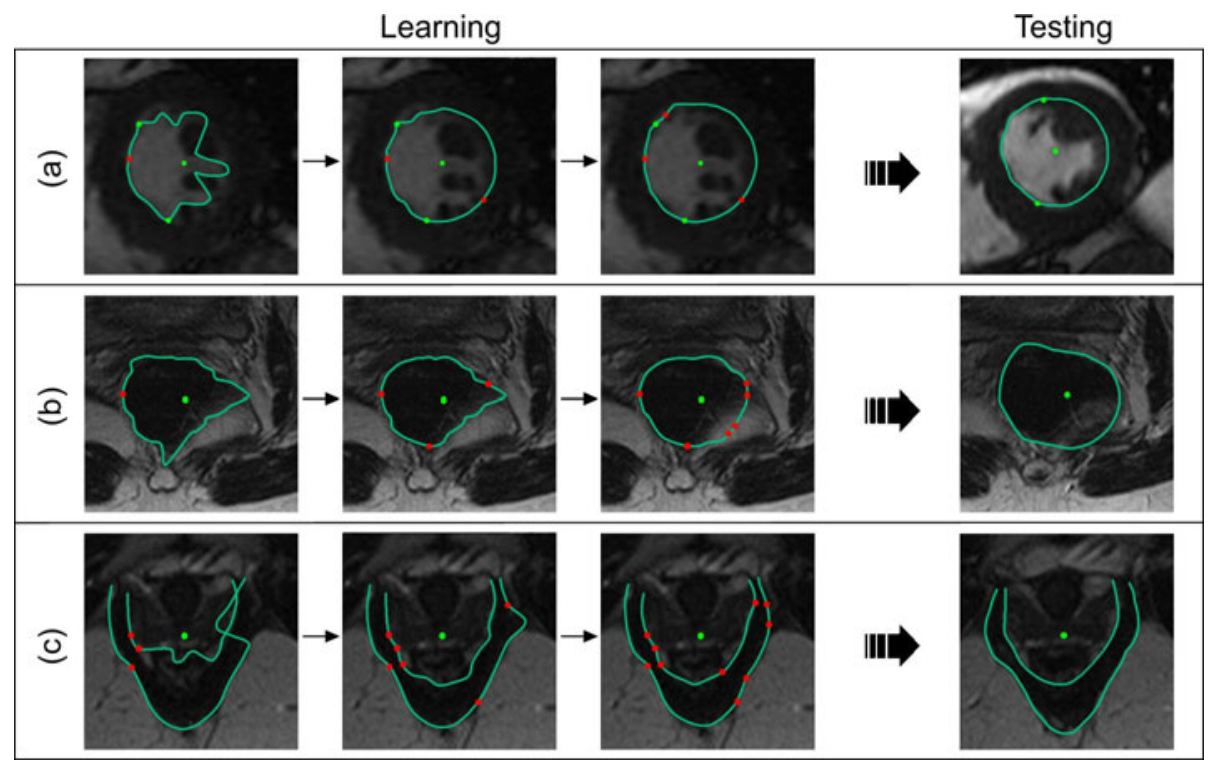

Fig. 1. Segmentation examples illustrating how user delineation or modification of the anatomical contours (shown in red) can be learnt through reinforcement learning and then used to perform context-specific segmentation: (a) the endocardial border of the left ventricle when the papillary muscles are excluded; (b) the boundary of the rectum showing how to adapt to the partial volume effect in MR; (c) the inner and outer contours of the pelvic floor when both subtle and strong appearance features are present. It is worth noting that the algorithm learns to extrapolate the shape when there are no clear image appearance features to rely upon. Under the proposed reinforcement learning framework, the predicted boundary points get either reward or punishment through user acceptance (implicit) or correction (explicit), thus making the segmentation model context specific and generalizable to unseen geometrical variations.

The purpose of this paper is to propose a reinforcement learning framework for context aware segmentation and to demonstrate how user-specific behavior can be assimilated in-situ for effective model adaptation and learning. Central to the proposed technique is a two-layer reinforcement learning algorithm that builds the model from accumulated experience during user interaction. One advantage of the framework is that the learning process circumvents the need for extensive off-line training, and thus is readily generalizable to unseen pathological patient cases in a context or subject specific manner. As the algorithm learns 'pervasively' when the user performs manual segmentation, no additional steps are required for the training process. Furthermore, the method is able to adapt and improve its accuracy as experience is acquired, a process that is akin to the training of human observers. The example results in Fig. 1 demonstrate the potential of the technique. In this paper, we use left ventricular segmentation from MR images to illustrate the overall structure of the rein- 
forcement learning framework and assess the extent to which the level of user interaction can be reduced, thus leading to an efficient semi-automatic segmentation approach.

\section{Methods}

\subsection{Two-Layer Reinforcement Learning}

Reinforcement learning [7] is a process where the learning agent interacts with the environment and tries to maximize its expected long-term reward. It is particularly suited to problems where explicit knowledge for solutions is difficult to obtain. By interacting with the user, the learning agent is not informed explicitly how to combine different strategies to attain the correct contour point. Instead, with the example given by the user, the agent only knows the level of its own performance. In general, there are three key elements of reinforcement learning, namely: state, action and reward. A state is a situation of the environment. An action is some behavior of the learning agent that interacts with the environment. A reward is the feedback that the agent receives from the environment during interaction, and in the case of image segmentation, the quality of localizing anatomical structures. The expected long-term reward of a given state or state-action pair is referred to as a value.

Q-learning [8] is a widely used reinforcement learning algorithm, where a table is maintained for recording values of all the possible states or state-action pairs. For image segmentation, however, due to the diversity of both geometrical and appearance information present within the image, inevitably there tend to be many states and actions for the learning agent; therefore the standard Q-learning approach in this case can be problematic. One way to solve this problem is to use value function approximation. To perform the approximation, parameters with much lower dimensionality than the actual state-action space are used to describe the value function. With these parameters, the agent does not attempt to find the best action for a given state directly as in Q-learning. Instead, it estimates the parameters that maximize the value function, which correspond to the best action. The estimation can be performed by minimizing the mean square error over the observed examples. For the minimization process, a policy gradient approach can be used. This adjusts the parameter vector during trials along the direction where the estimation error is minimized for the given example:

$$
\vec{\theta}_{t+1}=\vec{\theta}_{t}+\gamma\left[V^{\pi}\left(a_{t}\right)-V_{t}\left(a_{t}\right)\right] \nabla_{\theta} V_{t}\left(a_{t}\right)
$$

where $\vec{\theta}$ is the parameter vector, $t$ is the step index, $V^{\pi}\left(a_{t}\right)$ is the true value of the action $a_{t}, V_{t}\left(a_{t}\right)$ is the estimate value at step $t$, and $\gamma$ is a constant coefficient.

For a typical segmentation task, the three elements of the reinforcement learning framework can be defined as follows: a state is the appearance (e.g. the intensities along a given search direction); an action for a state is to localize a point on the anatomical border; and the reward is defined as an evaluation function. The parameters for segmentation are the weightings of different candidate strategies used to localize contour points. 
For the purpose of left-ventricular segmentation, candidate strategies include an Akima spline [9] as the basic geometrical representation of the anatomy due to its resilience to outliers. For simplicity, we have adopted a radial search strategy commonly used for LV segmentation. By combining both appearance and geometry information, the cost function to localize a contour point on a given search direction can be defined as:

$$
f(x, \alpha, \vec{w} \mid I)=\alpha\left(\sum_{i=1}^{n} w_{i} f_{A i}(x \mid I)\right)+(1-\alpha) f_{G}(x)
$$

where $x$ is the pixel position on the radial, $I$ is the intensity vector, $f_{A i}(x)$ is the $i$ th strategy within the appearance strategy category, $w_{i}$ is weighting for $f_{A i}(x)$, or referred to as the $i$ th in-category weighting, $\alpha$ is the weighting for the appearance strategy category, or referred to as cross-category weighting, $f_{G}(x)$ is the cost function for the geometry strategy, the weighting vector $\vec{w}=\left(w_{1}, w_{2}, \ldots, w_{n}\right)^{T}$ and $\sum_{i=1}^{n} w_{i}=1$.

The candidate contour point is defined by minimizing Eq. (2). This is denoted by $x_{\min }$ and is a function of weightings. Given an arbitrary set of weightings, $x_{\min }$ may not be optimum in terms of segmentation accuracy. The distance to the user example's position on the same radial can be used for evaluating $x_{\min }$ :

$$
g\left(\alpha, \vec{w} \mid I, f_{A 1}, \ldots f_{A n}, f_{G}\right)=-\left|x_{\min }\left(\alpha, \vec{w} \mid I, f_{A 1}, \ldots f_{A n}, f_{G}\right)-x_{T}\right|
$$

where $x_{T}$ is the example position. Therefore the learning task is, for every example, to optimize Eq. (3) with respect to the weightings. After learning, the optimal weightings are obtained for explicitly learnt samples, typically via user interaction.

In this paper, learning is separated into two layers, i.e., in-category weighting (i.e., $\vec{w}$ ) learning for appearance strategies, and cross-category weighting (i.e., $\alpha$ ) learning for appearance and geometry categories. This corresponds to a two-stage process, i.e., firstly to use appearance to localize the clear contour areas, and if it fails, then to combine geometry to interpolate the shape. Hence the evaluation function represented by Eq. (3) can be sub-divided into the following equations:

$$
\begin{gathered}
g_{\text {in }}\left(\vec{w} \mid I, f_{A 1}, \ldots f_{A n}\right)=-\left|x_{\min }^{\prime}\left(\vec{w} \mid I, f_{A 1}, \ldots f_{A n}\right)-x_{T}\right| \\
g_{\text {cross }}\left(\alpha \mid \vec{w}, I, f_{A 1}, \ldots f_{A n}, f_{G}\right)=-\left|x_{\min }\left(\alpha \mid \vec{w}, I, f_{A 1}, \ldots f_{A n}, f_{G}\right)-x_{T}\right|
\end{gathered}
$$

Eq. (4) is the evaluation of the localized point using appearance strategies only $\left(x_{\min }^{\prime}\right)$, whereas Eq. (5) is the evaluation of using both appearance and geometry strategies.

In the context of in-category weighting learning, the parameters for function approximation are the weightings for different appearance-based strategies. Meanwhile, because every learning episode for point localization on a radial has only one step, the immediate reward is the same as the expected long-term reward. Thus by replacing 
$\theta$ with $\vec{w}, V^{\pi}\left(a_{t}\right)$ with $g_{\text {in }}$ from Eq. (4) and $V_{t}\left(a_{t}\right)$ with 0 (since the learning agent expects its current action has zero error), Eq. (1) becomes

$$
\vec{w}_{t+1}=\vec{w}_{t}+\gamma\left|\left(g_{i n}(\vec{w})\right)_{t}\right| \nabla_{\vec{w}}\left(g_{i n}\right)_{t}\left(\vec{w}_{t}\right)
$$

In practice, the parameter update is realized as follows: when an example contour point is given by the observer, the algorithm regards it as the ground truth position on the contour; it then calculates the local gradient of the in-category weightings of appearance strategies and updates the weightings using Eq. (6), trying to minimize the difference between its localization and the user example.

For cross-category weighting learning, the weighting update equation is similar to (6) using policy gradient:

$$
\alpha_{t+1}=\alpha_{t}+\gamma\left|\left(g_{\text {cross }}(\alpha)\right)_{t}\right| \nabla_{\alpha}\left(g_{\text {cross }}\right)_{t}\left(\alpha_{t}\right)
$$

However, the learning flow for the cross-category weighting is significantly different due to two reasons. Firstly, unlike $f_{A i}$ in Eq. (4) which are independent from each other, $f_{G}$ in Eq. (5) depends on the appearance strategies, i.e., $f_{A i}$ and $\vec{w}$. This dependency is due to the design that the geometry strategy does not always rely on the user to provide control points. Instead, according to the learning agent's current knowledge, radials on which the appearance strategy category has a weighting large enough (i.e., $\alpha$ above a pre-defined threshold) use only the appearance category to localize the contour points, and these points becomes control points for the spline. Secondly, as the user examples directly provide positions as control points, $f_{G}$ depends on the positions of the user examples (by contrast all $f_{A i}$ are independent from the user input). Therefore, to perform policy gradient in such a dependent situation, an iterative flow is used: after the weightings on a new example radial are learned, they are used together with the example's position to update weightings of every previous example radial in the same image; then the updated weightings of previous example radials are used to update the weightings of the latest example. The iterative procedure continues until the weightings on example radials remain unchanged.

With the two-layer reinforcement learning, optimal weightings are learnt for radials in the appearance feature space where examples are available from the user. For segmenting an image, weightings on all radials need to be estimated based on the learned knowledge. The estimation is realized through Kriging [10], which interpolates the value of a random field.

\subsection{Validation and Data Collection}

The MR LV data used for validation were from a database of 56 subjects acquired from a 1.5T MR scanner (Siemens Sonata 1.5T, Erlangen, Germany) and a trueFISP sequence (in-plane pixel resolution $=1.1-2 \mathrm{~mm}$, slice thickness $=7 \mathrm{~mm}$ ). For deriving the ground truth data, manual delineation was performed by two expert clinicians using CMRtools (Cardiovascular Imaging Solutions Ltd., London, UK). 


\section{Results}

While Fig. 1 shows some examples of applying the proposed method to different structures, detailed validation is carried out on 3D LV endocardial borders of the enddiastolic frame and the end-systolic frames for the 56 subjects. For segmenting each slice, the user is required to provide three anchor points to initialize the algorithm: the centre of the LV blood pool and the RV/LV junctions on the endocardial border. The $\mathrm{RV} / \mathrm{LV}$ junctions and the points for corrections provided by the user are both used as training examples. The candidate strategies used in the appearance category include the maximal derivative along the radial, a 1D intensity profile and a 1D gradient profile, as they are popularly used in detecting endocardial borders. The strategies as well as the parameterization used are intended as an example for the reinforcement learning framework. For different applications, the reader may use other specific features, searching and parameterization techniques by switching to the corresponding stateaction space while maintaining the general reinforcement learning framework.

Table 1. The accuracy (in $\mathrm{mm}$ ) and the user input required by the proposed method and manual segmentation using CMRtools for segmenting the end-diastolic (ED) and the end-systolic (ES) frames (The step size is 0.2 for gradient calculation, $\gamma=1$ and the threshold of $\alpha$ is set to 0.9)

\begin{tabular}{llllll}
\hline \multicolumn{2}{l}{ Proposed Method } & \multicolumn{2}{l}{ Manual Segmentation } & Improvement \\
\hline Frame \& & Error & $\begin{array}{l}\text { Avg. No. of } \\
\text { Location }\end{array}$ & $\begin{array}{l}\text { Inter-Observer Va- } \\
\text { User Points }\end{array}$ & $\begin{array}{l}\text { Avg. No. of } \\
\text { riability of Accuracy }\end{array}$ & $\begin{array}{l}\text { User Interac- } \\
\text { User Points }\end{array}$ \\
\hline ED-Base & $0.81 \pm 0.25$ & 3.1 & $1.15 \pm 0.27$ & 7.0 & $55.7 \%$ \\
ED-Mid & $0.86 \pm 0.24$ & 3.5 & $1.00 \pm 0.33$ & 6.9 & $49.3 \%$ \\
ED-Apex & $0.70 \pm .23$ & 3.1 & $1.52 \pm 0.73$ & 6.6 & $53.0 \%$ \\
ED-All & $0.81 \pm 0.14$ & 19.6 & $1.18 \pm 0.34$ & 40.9 & $52.1 \%$ \\
\hline ES-Base & $0.67 \pm 0.22$ & 2.7 & $1.23 \pm 0.47$ & 5.4 & $50.0 \%$ \\
ES-Mid & $0.74 \pm 0.25$ & 3.1 & $1.08 \pm 0.59$ & 5.9 & $47.5 \%$ \\
ES-Apex & $0.53 \pm 0.20$ & 2.7 & $1.49 \pm 0.63$ & 5.1 & $47.1 \%$ \\
ES-All & $0.67 \pm 0.17$ & 17.5 & $1.27 \pm 0.45$ & 33.1 & $47.1 \%$ \\
\hline
\end{tabular}
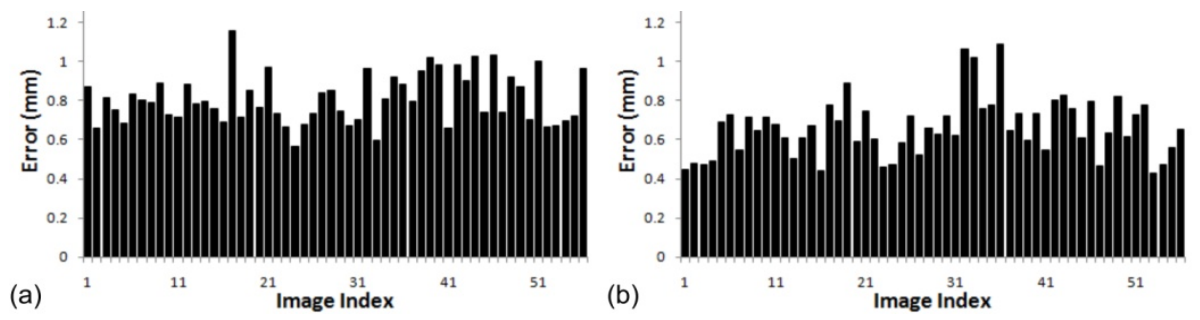

Fig. 2. Detailed segmentation results for the end-diastolic (a) and the end-systolic frame (b)

The segmentation result is compared with the manual segmentation for both accuracy and the number of user input points required, as summarized in Table 1. It is evident that for both the end-diastolic and the end-systolic frames, the amount of user interaction is significantly reduced whilst maintaining the overall accuracy as shown in Fig. 2. The paired t-test shows that the improvement is significant for both frames 
with $p \ll 0.0001$. It is worth noting that the result of the proposed method has an error much smaller than the inter-observer variability. The reason is that in cardiac $\mathrm{MR}$, segmentation is not just dependent on geometrical features or appearance information (e.g. dealing with papillary muscle), but also other prior knowledge, which leads to higher inter-observer variability. The ability through reinforcement learning to acquire this specific knowledge minimizes the error involved, and thus gives improved consistency. More error metrics are summarized in Table 2.

Table 2. The Jaccard index and the maximal radial difference of the proposed method

\begin{tabular}{lllll}
\hline & End-Diastole & \multicolumn{3}{l}{ End-Systole } \\
\hline Location & Jaccard Index & Max. Radial Diff. & Jaccard Index & Max. Radial Diff. \\
\hline Basal & $0.933 \pm 0.022$ & $2.55 \mathrm{~mm}$ & $0.915 \pm 0.031$ & $2.41 \mathrm{~mm}$ \\
Middle & $0.928 \pm 0.019$ & $2.60 \mathrm{~mm}$ & $0.906 \pm 0.036$ & $2.49 \mathrm{~mm}$ \\
Apical & $0.926 \pm 0.025$ & $2.04 \mathrm{~mm}$ & $0.889 \pm 0.048$ & $2.00 \mathrm{~mm}$ \\
All & $0.929 \pm 0.012$ & $2.60 \mathrm{~mm}$ & $0.905 \pm 0.030$ & $2.49 \mathrm{~mm}$ \\
\hline
\end{tabular}
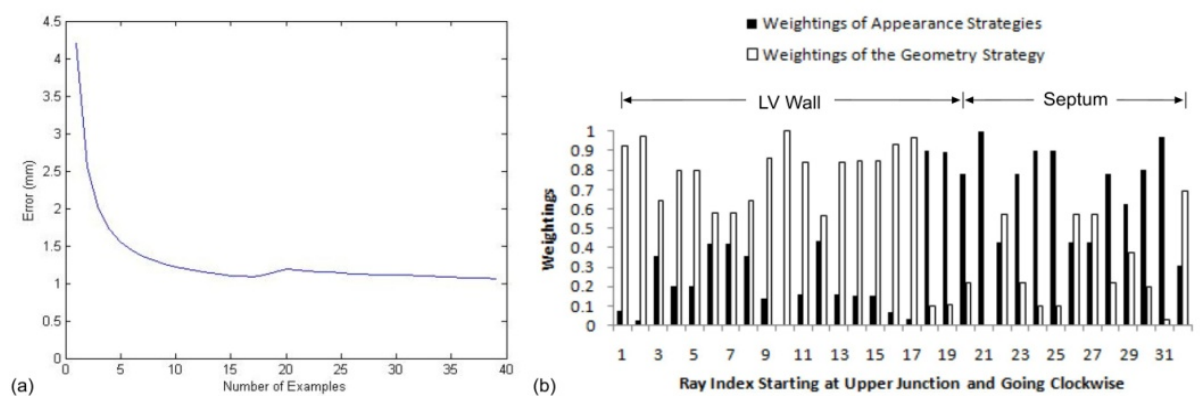

Fig. 3. (a) Learning curve; (b) Learned cross-category weightings for radials in a slice

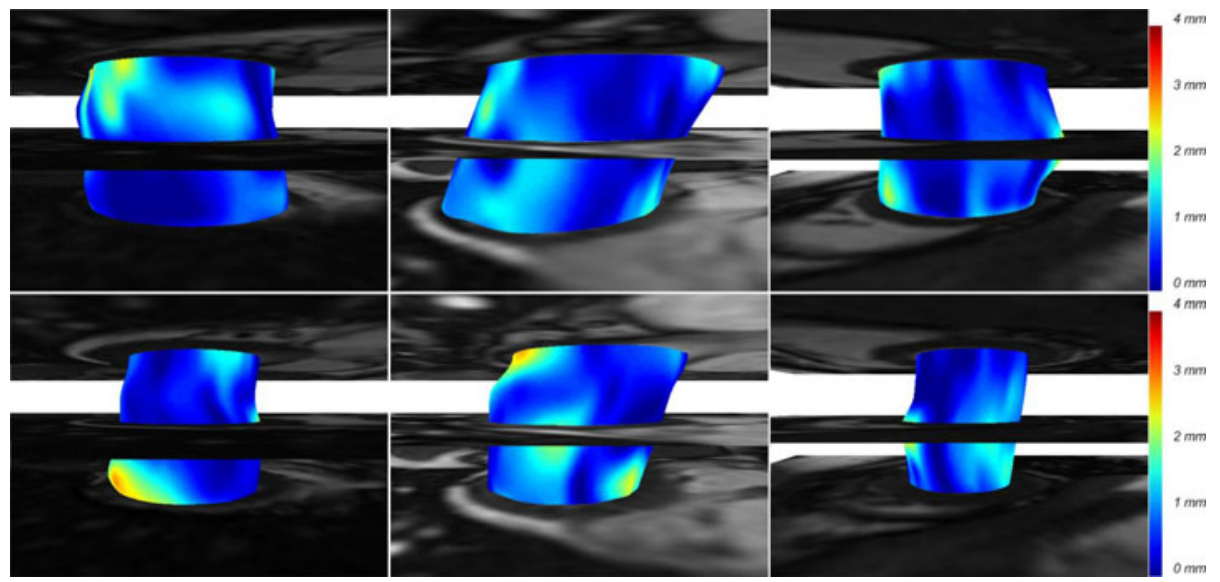

Fig. 4. Three instances of the end-diastolic (top) and end-systolic (bottom) frames showing surface localization errors. High accuracy is achieved with the proposed algorithm. 
Further analysis is carried out to evaluate the reinforcement learning process. A fixed data set of $203 \mathrm{D}$ cardiac MR images at the end-systolic frame is used for testing the learning behavior, while the rest of the data are used to interact with the user as in the experiment above. By using the proposed method, the model is updated whenever a new user example is given. The updated model is then used to segment all the testing images without correction. The results are shown in Fig. 3 (a). It is clear that with an increasing number of examples, the model is able to perform the segmentation more accurately. Fig. 3 (b) shows the cross-type weightings derived from the model for all the points around the endocardial border for a mid-ventricular slice at end-systole. As expected, it can be seen that at the LV wall region, the segmentation relies more on the geometry strategy, while at the septal region it relies more on the appearance strategies. Fig. 4 illustrates three examples of the diastolic and systolic volumes of the LV, highlighting regional errors involved in the segmentation results.

\section{Conclusion}

In this paper, we have presented a general semi-automatic scheme that learns from the user behavior to perform medical image segmentation. The algorithm determines the weightings of different segmentation strategies adaptively, which otherwise need to be either empirically defined or obtained from off-line training, and thus cause overfitting or poor generalization. The adaptation is achieved by a two-layer reinforcement learning algorithm that learns from the user examples. Detailed validation on MR LV segmentation demonstrates the potential clinical value of the algorithm.

\section{References}

1. Xu, C., Prince, J.L.: Snakes, shapes, and gradient vector flow. IEEE Transactions on Image Processing 7, 359-369 (1998)

2. Chan, T., Vese, L.: Active contour without edges. IEEE Transactions on Image Processing 10, 266-277 (2001)

3. Cootes, T.F., Hill, A., Taylor, C.J., Haslam, J.: The use of active shape models for locating structures in medical images. In: Barrett, H.H., Gmitro, A.F. (eds.) IPMI 1993. LNCS, vol. 687, pp. 33-47. Springer, Heidelberg (1993)

4. Wang, Y., Staib, L.H.: Boundary finding with prior shape and smoothness models. IEEE Transactions on Pattern Analysis and Machine Intelligence 22, 738-743 (2000)

5. Davatzikos, C., Tao, X., Shen, D.: Hierarchical active shape models, using the wavelet transform. IEEE Transactions on Medical Imaging 22, 414-423 (2003)

6. Lekadir, K., Keenan, N.G., Pennell, D.J., Yang, G.-Z.: An inter-landmark approach to 4-D shape extraction and interpretation: application to myocardial motion assessment in MRI. IEEE Transactions on Medical Imaging 30, 52-68 (2011)

7. Sutton, R.S., Barto, A.G.: Reinforcement learing: an introduction. The MIT Press, Cambridge (1998)

8. Watkins, C.J.C.H.: Learning from delayed rewards. PhD Thesis, Cambridge University (1989)

9. Akima, H.: A method of smooth curve fitting (1969)

10. Matheron, G.: Principles of geostatistics. Economic Geology 58, 1246-1266 (1963) 